\title{
In silico biosynthesis of virenose, a methylated deoxy-sugar unique to Coxiella burnetii lipopolysaccharide
}

\author{
Gabriela Flores-Ramirez', Stefan Janecek², Ján A Miernyk ${ }^{3,4,5}$ and Ludovit Skultety ${ }^{1,6^{*}}$
}

\begin{abstract}
Background: Coxiella burnetii is Gram-negative bacterium responsible for the zoonosis Q-fever. While it has an obligate intracellular growth habit, it is able to persist for extended periods outside of a host cell and can resist environmental conditions that would be lethal to most prokaryotes. It is these extracellular bacteria that are the infectious stage encountered by eukaryotic hosts. The intracellular form has evolved to grow and replicate within acidified parasitophorous vacuoles. The outer coat of C. burnetii comprises a complex lipopolysaccharide (LPS) component that includes the unique methylated-6-deoxyhexose, virenose. Although potentially important as a biomarker for $C$. burnetii, the pathway for its biosynthesis remains obscure.

Results: The 6-deoxyhexoses constitute a large family integral to the LPS of many eubacteria. It is believed that precursors of the methylated-deoxyhexoses traverse common early biosynthetic steps as nucleotidemonosaccharides. As a prelude to a full biosynthetic characterization, we present herein the results from bioinformatics-based, proteomics-supported predictions of the pathway for virenose synthesis. Alternative possibilities are considered which include both GDP-mannose and TDP-glucose as precursors.

Conclusion: We propose that biosynthesis of the unique C. burnetii biomarker, virenose, involves an early pathway similar to that of other C-3'-methylated deoxysugars which then diverges depending upon the nucleotide-carrier involved. The alternatives yield either the D- or L-enantiomers of virenose. Both pathways require five enzymatic steps, beginning with either glucose-6-phosphate or mannose-6-phosphate. Our in silico results comprise a model for virenose biosynthesis that can be directly tested. Definition of this pathway should facilitate the development of therapeutic agents useful for treatment of $\mathrm{Q}$ fever, as well as allowing improvements in the methods for diagnosing this highly infectious disease.
\end{abstract}

Keywords: Coxiella burnetii, LPS, Deoxysugars, Virenose, Biosynthetic pathway

\section{Background}

Coxiella burnetii, the causative agent of $\mathrm{Q}$ fever in humans, is a highly infectious intracellular bacterium that resides in the parasitophorous vacuole of host cells [1]. It causes several outbreaks of this zoonotic disease each year $[2,3]$. Infected livestock are mainly asymptomatic, but under certain circumstances display infertility, endometritis, placentitis, abortions, stillbirth, and delivery

\footnotetext{
* Correspondence: viruludo@savba.sk

'Department of Rickettsiology, Institute of Virology, Slovak Academy of Sciences, Dubravska cesta, 9, Bratislava 845 05, Slovakia

${ }^{6}$ Centre for Molecular Medicine, Slovak Academy of Sciences, Bratislava 831 01, Slovakia

Full list of author information is available at the end of the article
}

of weak offspring [4-6]. Human Q fever generally results from inhaling infectious aerosols produced by domestic animals, can be either acute or chronic, and exhibits a wide spectrum of clinical manifestations [7-11].

Coxiella have an extracellular matrix similar to that of other Gram-negative bacteria. The outer coat of virulent phase I C. burnetii isolates, from natural sources or infections, is critical to evading the host immune system and include full-length lipopolysaccharides (LPS). It includes an $\mathrm{O}$-antigen containing two unique sugars, virenose (6-deoxy-3-C-methyl-D-gulose) and dihydrohydroxystreptose (3-C-(hydroxymethyl) lyxose). These sugars have been used as biomarkers of phase I $C$. burnetii cells and are not present in phase II [12-17]. Serial

\section{Biomed Central}


in vitro passage of $C$. burnetii in either embryonated hen eggs or tissue culture results in cells with morphologically, structurally, and compositionally different from phase I $[18,19]$. These changes accompany a chromosomal deletion which corresponds to one of the clusters in the genome necessary for O-antigen biosynthesis $[20,14]$. Two clones, clone I (9Mi/II/C1) and $4(9 \mathrm{Mi} / \mathrm{II} / \mathrm{C} 4)$ of the Nine Mile strains classified as avirulent phase II, have a genomic deletion of 25,997 bp and the third isolate, 9Mi/Baca, which was derived from $9 \mathrm{Mi} / \mathrm{I}$ by passing for 4,091 days in cell cultures, has a shorter deleted region [20]. The results from sequence analyses indicate that a group of LPS-biosynthetic genes, including genes that encode epimerases, dehydratases, and nucleotide-sugar glycosyltransferases, are part of the deleted segment [14,20].

In our recent comparative proteomics study of phase I and phase II of C. burnetii [21], seventeen proteins involved in LPS biosynthesis and metabolism were identified. Nine of these were detected in phase I but not in phase II cells, and are products of genes located in the deleted region of the chromosome. Thus, we confidently proposed these virulence-associated proteins are related to biosynthesis of the LPS I biomarkers. Although, virenose was found to be D-gulo enantiomer with the $4 \mathrm{C} 1$ ring conformation by NMR spectroscopy [16], the L-form with $1 C 4$ conformation have been reported previously as well [17]. This potential ambiguity prompted us to examine the possible pathways for biosynthesis of the both enantiomers. Herein we propose a homology-based biosynthetic pathway for virenose based upon bioinformatic analyses and supported by the results from prior genomic and proteomic analyses.

\section{Results and discussion}

The 6-deoxysugars constitute a large family of essential components of the LPS of many eubacteria [22]. They are produced by biosynthetic pathways which share early steps [23,24]. During biosynthesis, the monosaccharideprecursors are activated to one of four nucleosidediphosphate carriers (NDP); adenosine diphosphate (ADP), thymidine diphosphate (TDP), guanosine diphosphate (GDP), or uridine diphosphate (UDP) [22,25] that are then exchanged in the final step by an enzyme which transfers the sugar to a lipid carrier, forming the $\mathrm{O}$ polysaccharide unit. The 6-deoxyhexoses can be synthesized from D-glucose-6-phosphate which is a precursor for the biosynthesis of the TDP, CDP and UDP-sugars, or fructose-6-phosphate which is converted to mannose6-phosphate and serves as precursor of the GDP-sugars $[22,23,25]$. The activated precursor NDP-4-keto-6-deoxyalpha-D-hexose is then transformed by various enzymatic modifications (e.g., epimerization, C- and O-methylation, deoxygenation, amination, ketoreduction, acetylation, dehydrations, etc.) to a great variety of deoxysugars [22,23].

\section{Glucose-6-phosphate and fructose-6-phosphate as the initial precursors of virenose}

The pathway for virenose synthesis depends upon of its enantiomeric and ring conformations. One version involves activation of glucose using TDP, while in the second mannose is activated using GDP. In either instance, the hexose is phosphorylated at $\mathrm{C} 1$ before their activation by a nucleotidylyltransferase.

There is biochemical evidence that $C$. burnetii can convert glucose to pyruvate [26-28], however, genome sequence analysis of all six isolates has thus far failed to identify a hexokinase responsible for converting glucose to glucose-6-phosphate (Figure 1) or glucose-6-phosphate and 6-phosphogluconate dehydrogenases [29]. Thus, the first steps of both glycolysis and the pentose phosphate pathway appear missing [29]. This might well explain the low biosynthetic capacity and slow growth rate observed for C. burnetii. We speculate that C. burnetii phosphorylates glucose via a transphosphorylation reaction involving carbamoyl-phosphate and a phosphatidic acid phosphatase family protein encoded by CBU_1267, as described for the $9 \mathrm{Mi} / \mathrm{I}$ isolate [29]. There are, of course, other as yetpoorly defined alternatives. Possibly glucose-6-phosphate (2) is obtained from the host cells. Both GDP-mannose, and fructose 6-phosphate (1) or mannose-6-phosphate (3) are potential sources of glucose-6-phosphate, invoking participation of a mannose-6-phosphate isomerase pyrophosphorylase-type or reaction (PMI-GMP; E.C. 5.3.1.8) [30] such as that found as a participant in synthesis of the capsular polysaccharide of Pseudomonas aeruginosa, Salmonella thyphimurium, and Xanthomonas campestris [31,32]. The PMI-GMP enzymes posses separate domains for the mannose isomerase (PMI) and GDP-D-mannose pyrophosphorylase (GMP) activities [33]. A zinc-binding motif and the catalytic amino acid residue R408 are both characteristic of PMI activity [34]. The GMP activity is defined in the N-terminal by the pyrophosphorylase signature sequence, GXGXR(L)-PK [34]. Based on sequence analysis and comparison to C. burnetii genome, the PMIGMP activity might be catalyzed by the product of the CBU_0671 gene, which includes both of these signatures. It shares $45 \%$ (E value e-113), 46\% (E value $3 \mathrm{e}-122$ ), and $39 \%$ amino acid identity (E value $4 \mathrm{e}-88$ ) with the PMIGMP from Salmonella enterica LT2 (AAG41744.1), Escherichia coli (YP_002413091), and Helicobacter pylori (YP_626781) (Additional file 1-A).

\section{Phosphohexomutase}

Both glucose-6-phosphate (2) and mannose-6-phosphate (3) are transformed to the corresponding hexose-1phosphate by a phosphohexomutase [22,23,25] during biosynthesis of deoxysugars. Phosphomannomutase/phosphoglucomutase (PMM/PGM; E.C. 5.4.2.8) can convert both glucose and mannose, which are $\mathrm{C} 2$ epimers. In $C$. 


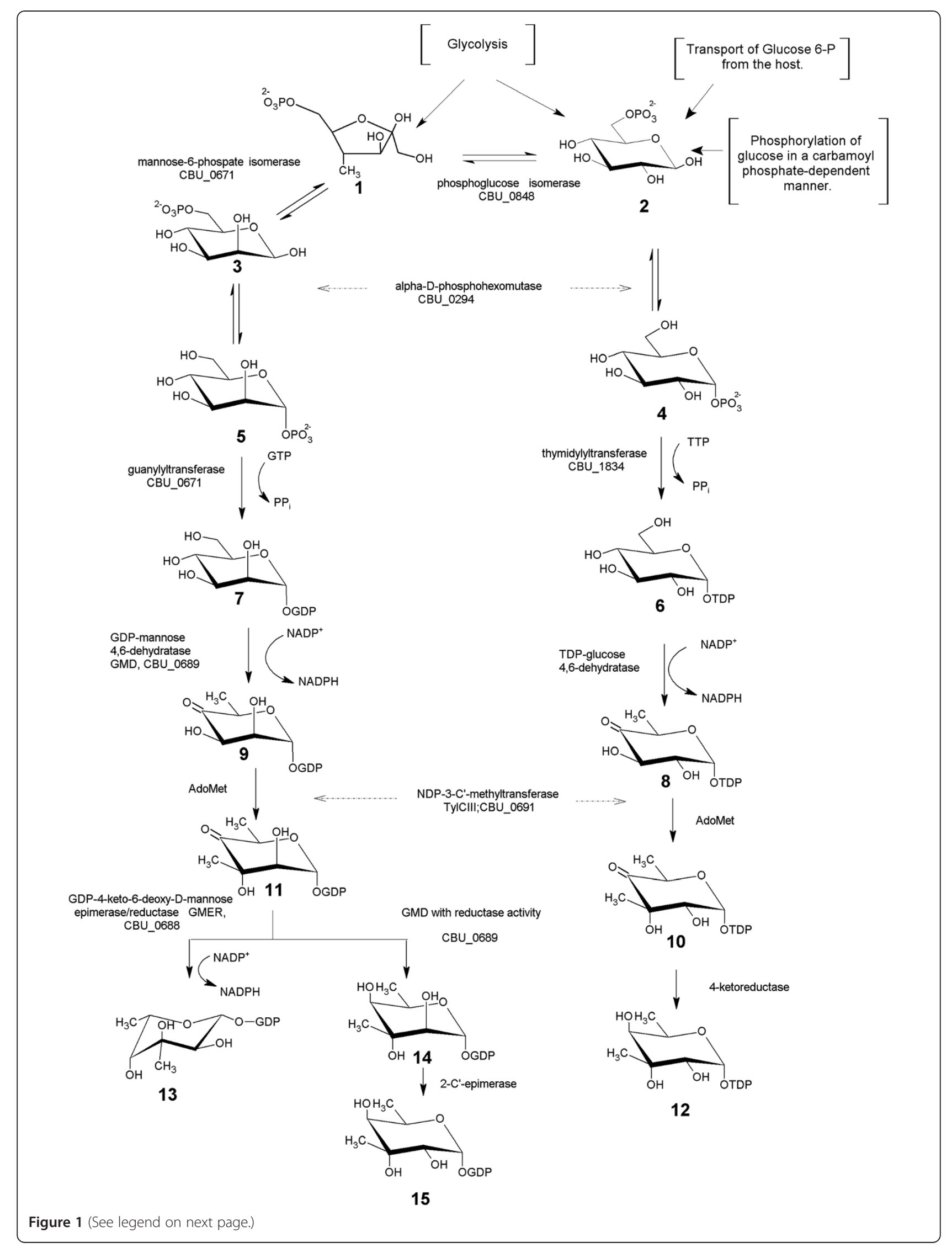




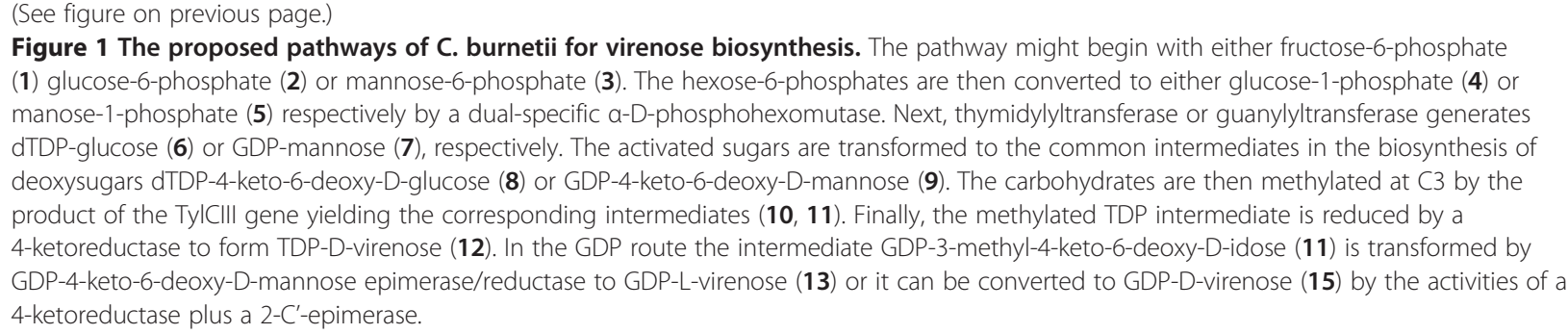

burnetii, the product of the CBU_0294 locus has 53\% sequence identity (E value e-145) with the PMM/PGM from $P$. aeruginosa (PDB: 1K2Y)[35] (Additional file 1-B). It catalyzes a reversible intramolecular phosphoryl-transfer from C6 to C1 using a Ser108 phospho-enzyme intermediate [35]. The PMM/PGM sequence of $P$. aeruginosa includes the sugar binding motif GEMS(G/A) at positions 324-328 (Additional file 1-B), the catalytic residue is R421, and residues involved in phosphate binding (Y17, K285, R421, S423, N424, and T425) [35]. All of these sequence motifs are conserved in the PMM/PGM sequence of $C$. burnetii (CBU_0294).

\section{Nucleotide-sugar formation}

Both glucose-1-phosphate (4) and mannose-1-phosphate (5) would need to be activated to a NDP-derivate. The activation of D-glucose-1-phosphate (4) to dTDP-Dglucose (6) would employ D-glucose-1-phosphate thymidylyltransferase (G1PTT; EC 2.7.7.24)[36], which has been demonstrated to be involved in the synthesis of Lrhamnose, a common component of the cell wall of many pathogenic bacteria. The $P$. aeroginosa enzyme has been named RmlA (AAG08548) [37], while the Salmonella typhi enzyme was designated RfbA (NP_456644) [38]. In the actinobacteria, G1PTT is referred to as TylA1 (AAA2134), and is associated with the synthesis of mycarose, a component of the antibiotic tylosine produced by Streptomyces fradiae [36] or eryA when associated with synthesis of erytromycin by Saccharopolyspora erythraea [39]. Based on sequence analysis, the corresponding C. burnetii gene product (CBU_1834) shares 62\% (E value e-105), 61\% (E value e-101), and 63\% (E value 4e-107) amino acid identity with $\mathrm{RfbA}, \mathrm{RmlA}$, and TylA1, respectively. The primary structure of the protein encoded by the G1PTT from $P$. aeroginosa contains the motif (G)GXGXR(L) and the catalytic residues R15, K25, D110, K162 and D225, as well as residues involved in the specificity for thymidine, G10, Q82, and G87, which form hydrogen bonds with the pyrimidine ring. All these residues are conserved in various G1PTT [38] as well as in the product of the CBU_1834 gene (Additional file 1-C), although CBU_1834 is located outside of the $\mathrm{O}$-antigen gene cluster region. It has, however, been previously reported that enzymes integral to specific pathways are often located outside of otherwise common genic-regions [40].

If the pathway includes mannose-1-phosphate (5) as an intermediate, it can be transformed to GDP- $\alpha-D$ mannose (7) by mannose-1-phosphate guanylyltransferase. Results from BLAST analyses indicate similarity with the product of CBU_0671 and the guanylyltransferase.

\section{NDP-4-keto-6-deoxy-hexose is the key intermediate}

After activation, any of the proposed intermediates will need to be transformed into the key precursor for all NDTP-sugars, an NDP-4-keto-6-deoxy-hexose. This transformation involves dehydratation at $\mathrm{C} 4$ and $\mathrm{C6}$ via dNDP-D-hexose 4,6-dehydratase activity [22]. The dTDPD-glucose (6) might be transformed into dTDP-4-keto-6deoxy-D-glucose (8) by the TDP-glucose 4,6 dehydratase (TGD). The product of RmlB (EC 4.2.1.46) from Salmonella enterica serovar thypimurium [41] was first identified in the L-rhamnose biosynthetic pathway. Homologues of this enzyme have also been found in Streptomyces venezuelae, E. coli K12, P. aeroginosa, Pasteurella pseudotuberculosis and plants [41]. Sequence analysis in C. burnetii revealed eight gene products sharing similarity with $\mathrm{RmlB}$ (PDB: 1KEU) of S. typhi. The extent of sequence identity is similar among all eight proteins, CBU_0677 (27\%; E value 2e-23), CBU_0844 (22\%; E value 4e-21), CBU_0689 (24\%; E value 2e-15), CBU_0829 (25\%; E value 1e-14), CBU_0676 (24\%; E value 8e-12), CBU_0688 (21\%; E value 1e-4), CBU_1837 (22\%; E value 2e-4) and CBU_0681 (21\%; E value 0.015) (Additional file 1-D). Both CBU_0688 and CBU_0689 were located within the deleted multigenic region thought to be necessary for the synthesis of LPS. At the same time, CBU_0676 and CBU_0677 are close to this region. In contrast, CBU_1837 is near to the thymidylyl transferase gene (CBU_1834). The results from structural studies indicate that the protein is a homodimer that catalyzes a NAD-dependent reaction. The conserved catalytic triad includes YXXXK (residues 167-171 in RmlB) and a conserved motif GXXGXXG at the Nterminus. Both motifs, together with the His residue at position 300 which binds the ribose of dTDP, are conserved in the candidates sequences predicted to have dTDP D-glucose-4,6-dehydratase activity in C. burnetii. Moreover RmlB also exhibits both structural and mechanistic 
similarities to the other NDP-hexose-4,6-dehydratases. In toto, the conservation of characteristics makes it very difficult to make a bioinformatics-based prediction of which specific single gene product is involved in the pathway.

An identical reaction is required to transform the GDP$\alpha$-D-mannose (7) into GDP-4-keto-6-deoxy-D-mannose (9) employing the GDP-mannose-4,6-dehydratase (GMD) which activity was biochemically characterized in Mortierella alpine [42]. The structure of GMD (PDB: 1RPN) from $P$. aeroginosa was determined recently [43]. This enzyme is involved in the biosynthetic pathway leading to GDP-Drhamnose [43], a sugar found at trace levels in the LPS of $C$. burnetii [16]. The results from BLAST analyses indicate that CBU_0689 has a 51\% (E value e-105) and 50\% identity (E value 7e-91) to both GMD from E. coli (PDB; 1DB3) [44] and P. aeroginosa (PDB; 1RPN)[43], respectively (Additional file 1-E). This enzyme contains a characteristic Gly-rich fingerprint, G9-XR-G-XX-5, along with the catalytic residues T126, E128, Y150, and K154 (Additional file $1-E)$. In C. burnetii, this gene is present in the region deleted from the phase II genome. Furthermore, the diagnostic catalytic T126 is replaced with a Ser residue. However, the T126S change was also found in the GMD sequences from Aneurinibacillus thermophilus, Mycobacterium tuberculosis, and Methanobacterium thermoautotrophicum $[43,44]$.

\section{Methylation}

Because virenose has a C-3' methyl group, the key precursor NDP-4-keto-6-deoxy-hexose is also likely to be methylated. Among deoxysugars, methylation reaction at this position are mediated by NDP-hexose C-3'methyltransferases (TylCIII; E.C. 2.1.1.-) using S-adenosylmethionine (adoMet) as the methyl donor. A role for this enzyme has been demonstrated in the biosynthesis of L-mycarose, erythromycin, and avilamycin by S. fradiae [45], S. erythraea [46], and S. viridochromogenes [47], respectively. In C. burnetii, the product of the CBU_0691 locus has 44\% (E value 1e-127), 44\% (E value $3 e-121$ ) and $42 \%$ (E value 7e-119) amino acid identity with TylCIII from $S$. fradiae (AAD41823), EryBIII from S. erythrae (YP_001102998), and aviGI from S. viridochromogenes (AAK83176), respectively. All these proteins include consensus sequence regions which typify adoMet-dependent methyltransferases [47] (Additional file 1-F). Motif I is a 9-residue sequence with a conserved Gly positioned 5 residues from the N-terminus and an Asp located 17 residues after the C-terminus, and which mediates contact with adoMet [48]. The D-loop, or motif II, contains an acidic residue, either Asp or Glu, whose side-chain hydrogen makes a bond to the ribose-hydroxyl group of AdoMet [49]. Both motifs are present also in the sequence of the product of CBU_0691 of C. burnetii (Additional file 1-F). Although this enzyme has only been demonstrated to be active with
TDP-sugars, there is no a priori reason that it might not be able to accommodate a range of NDP-sugars. The results from mechanistic studies indicate that the methylation reaction catalyzed by TylCIII proceeds with an inversion of hydroxyl groups at C-3' [45]. Thus, the equatorial methyl group at C-3' position and the axial C-3' hydroxyl group that are structural features of D- (12) and Lvirenose (13) $[16,17]$ are reversed.

\section{Reduction and epimerization}

In the proposed biosynthetic pathway leading to TDPD-virenose (12), i.e., the isomer suggested by Toman and Skultety [16] to be present in the LPS of virulent phase I C. burnetii, only a reduction at C4 of TDP-3methyl-4-keto-6-deoxy-D-gulose (10) is necessary. Several 4-ketoreductases are potentially able to provide this reduction. They include the product of the tylCIV locus from S. fradiae (AAD41822.1) which is involved in biosynthesis of mycarose[50,51], the product of the mtmU gene from $\mathrm{S}$. argillaceus associated with D-oliose and Dolivose biosynthesis [52,53], and StrL which participates in the synthesis of streptomycin [54]. While the sequences of homologues of 4-ketoreductases are broadly conserved, we were unable to find any obvious candidates within the C. burnetii proteome. Thus, we speculate that a dual specificity-enzyme is involved. Recently it was reported that the enzyme encoded by TylCIII (CAK50784.1) of $S$. argillaceus has 4-ketoreductase activity [53]. The $C$. burnetii protein encoded by the CBU_0691 locus has 30\% identity (E value 4e-49) to TylCIII and we propose that this is the relevant 4-ketoreductase.

In contrast, we have identified three different candidates for the 4-ketoreductase that would be involved in the synthesis of GDP-sugars. These include the GDP-4keto-6-deoxy-D-mannose reductase (RMD) that is involved in several pathways [55-58], the bifunctional GMD that catalyzes both 4,6-dehydratase and reductase reactions leading to GDP-D-rhamnose synthesis in Klebsiella pneumoniae, Anaurinibacillus thermoaerophilus, and P. aeruginosa [55,58], and the bifunctional GDP-4-keto-6-deoxy-Dmannose epimerase/reductase (GMER; E.C. 1.1.1.271), which is responsible for the last two steps of the GDP-Lfucose synthesis in E. coli [59] and M. alpine [42]. The pathway to GDP-L-virenose (13), i.e., the isomer suggested by Schramek et al. [17] to be present in the LPS of virulent phase I C. burnetii, requires both 4-ketoreductase and epimerase activities. The last intermediate, GDP-3-methyl-4keto-6-deoxy-D-idose (11), must undergo epimerization at positions $\mathrm{C}-3$ and $\mathrm{C}-5$, yielding the L-enantiomer, followed by a 4 keto-reduction catalyzed by a NADPH-dependent GMER [60]. In C. burnetii, the product of CBU_0688 shares $45 \%$ identity (E value $5 \mathrm{e}-78$ ) with the well-described dual-activity GMER from E. coli (PDB: 1E6U) [60]. The active site of this enzyme includes S107, Y136, and K140 
together with the catalytic residues $\mathrm{C} 109$ and $\mathrm{H} 179$ required for epimerization reaction [60]. These amino acids/ positions are conserved in the C. burnetii sequence (Additional file 1-G).

Although the GDP-sugar pathway to GDP-L-virenose appears more likely, there remains the possibility that GDP-D-virenose (15) is involved. Starting with the GDP3-methyl-4-keto-6-deoxy-D-idose (11), a bifunctional GMD could provide both the dehydration and 4ketoreduction steps leading to GDP-3-methyl-6-deoxyD-idose (14) [55]. Finally, a 2-C'-epimerase would be required to form GDP-D-virenose (15). It has not yet been possible during the course of our bioinformatics studies to identify a candidate for the latter enzyme within the proteome of $C$. burnetii.

Recently, Narasaki et al. [61] published the results from analysis of the first three steps of a pathway potentially leading to GDP- $\beta$-D-virenose biosynthesis; fructose6-phosphate to mannose-6-phosphate to mannose-1phosphate to GDP- $\beta$-D-mannose. The enzymes involved, phospho-mannose isomerase, phospho-mannose mutase, GDP-mannose pyrophosphorylase, GDP-mannose 4,6dehydratase, and C-3'methyltransferase, are also part of one branch of our proposed pathway. It is important to consider, however, that while consistent with and fully supporting our proposal these three steps are not unique to biosynthesis of either virenose or dihydrohydroxystreptose $[22,25]$.

\section{Conclusions}

Herein we propose that biosynthesis of the unique $C$. burnetii biomarker, virenose, involves a pathway similar to that of other C-3'-methylated deoxysugars. Two alternative routes are provided that differ primarily in the nucleotide-sugar involved. The alternatives yield either the D- or L-enantiomers of virenose. Both routes require five enzymatic steps, beginning with either glucose-6phosphate or mannose-6-phosphate. For the pathway starting with glucose-6-phosphate and ending with D-virenose, we propose involvement of $\alpha$-D-phosphohexomutase, thymidyltransferase, TDP-glucose-4,6-dehydratase, NDP-hexose-3-C-methyltransferase, and an enzyme with 4-ketoreductase activity that cannot be confidently predicted from analysis of the C. burnetii genome. Alternatively, starting with mannose-6-phosphate we predict the involvement of $\alpha$-D-phosphohexomutase, guanyltransferase, GDPmannose-4,6-dehydratase, NDP-hexose-3-C-methyltransferase, and GDP-4-keto-6-deoxy-D-mannose epimerase/ reductase, leading to L-virenose. Our in silico results comprise a model for virenose biosynthesis that is entirely plausible based upon the results of proteomic analyses and which can be directly tested. Definition of this pathway will facilitate the development of therapeutic agents useful for treatment of $\mathrm{Q}$ fever as well as allow improvements in the methods for diagnosing this highly infectious disease.

\section{Methods}

\section{Sequence analyses}

The protein sequences were retrieved from the UniProt knowledge database [62] and NCBI [63] using BLAST searches [64] with the PMI-GMP (NCBI:AAG41744.1), PMM/PGM (PDB:1K2Y), G1PTT (NCBI:AAG08548), GMD (PDB:1RPN), TGD (PDB:1KEU), TylCIII (NCBI:AAD41823), and GMER (PDB:1EGU) sequences as queries. Sequences were aligned using Clustal-W2 [65] on the European Bioinformatics Institute's server (http://www.ebi.ac.uk/). Manual adjustments were made in order to maximize similarities. Structures were retrieved from the Protein Data Bank (PDB) [66].

\section{Additional Files}

Additional file 1: Alignments of amino acid sequences of predicted enzymes implemented in the synthesis of D- and L-virenose. (A)

Mannose-6-phosphate isomerase pyrophosphorylase (PMI-GMP). The blue highlighting indicates the pyrophosphorylase signature sequence $(\mathrm{N}$ terminus) while the zinc-binding motif (C-terminus) is green. The R408 of the C-terminal Zn-binding motif is involved in catalysis (in red). (B) Dual phosphomannomutase/phosphoglucomutase (PMM/PGM). The residues involved in phosphate binding (Y17 K285 R421 S423 N424 T425) are in blue together with the phosphorylated S108 (according to the structure of $P$ aeruginosa PMM/PGM; (PDB: 1K2Y). The residues involved in sugar binding (GEMS) are green. (C) Glucose-1-phosphate thymydylytransferase (G1PTT). The residues participating in catalysis and those responsible for thymidine-specificity are red and yellow respectively. The conserved $\mathrm{N}$-terminal motif GXXXXL is green. (D) DTP-D-glucose-4,6-dehydratase (TGD). BLAST analysis against the C burnetii proteome using the TGD from $S$ enterica (PDB:1G1A) gives eight candidates for such activity: CBU_0677 NAD-dependent epimerase/dehydratase family protein; CBU_0844 capsular polysaccharide biosynthesis protein I; CBU_0689 GDP_ mannose-46-dehydratase; CBU_0829 NAD-dependent epimerase/ dehydratase family protein; CBU_0676 NAD-dependent epimerase/ dehydratase; CBU_0688 GDP-fucose synthetase; CBU_1837 putative NAD-dependent epimerase/dehydratase family protein; and CBU_0681 conserved hypothetical protein. The results from structural studies have shown that the latter protein is a homodimer in a NAD-dependent reaction. The conserved catalytic triad includes YXXXK (residues 167-171 in 1KEU) in light blue and a conserved motif GXXGXXG at the N-terminus in green are conserved in C burnetii. The $\mathrm{H} 300$ (yellow) which binds the ribose of dTDP is conserved in four of the sequence candidates in $C$ burnetii. (E) GDP-mannose-46 dehydratase (GMD). The characteristic Glyrich fingerprint sequence of GILFNHEGPXRGXXFVTRK which binds NADPH is highlighted in dark green inside this zone R185 is conserved in all GMD sequences previously characterized (yellow). The catalytic residues T126 Glu128 Y150 and K154 are highlighted in light red. The R35 and R43 residues in $P$ aeroginosa comprise the RR loop which participates in the tetrameric interface are indicated in blue. (F) NDP-hexose 3-C methyltransferase (TyICIII). The motif I characteristic of S-

adenosylmethionine (SAM)-dependent methyltransferases is highlighted in light green; the Gly residue of the SAM-binding site is yellow and the motif II sequence is highlighted in blue with emphasis on the conserved Asp residue in yellow. The Asp positioned 17 residues after motif I thought to be important in making a contact with SAM is shown in orange. (G) The bifunctional GDP-4-keto-6-deoxy-D-mannose epimerase/ reductase (GMER). The catalytic residues S107 Y136 and K140 are light green the residues involved in epimerization C109 and H179 are yellow. The characteristic N-terminal GXXGXXG motif is green. 


\section{Abbreviations}

G1PTT: D-glucose-1-phosphate thymidylyl transferase; GMD: GDP-mannose4,6-dehydratase; GMER: GDP-4-keto-6-deoxy-D-mannose epimerase/ reductase; LPS: Lipopolysaccharide; NDP: Nucleostide dieoxy phosphate; PMM/PGM: Phosphomannomutase/phosphoglucomutase; RMD: GDP-4-keto6-deoxy-D-mannose reductase; TGD: TDP-glucose 4,6 dehydratase: ADP: Adenosine diphosphate; TDP: Thymidine diphosphate; TyICIII: NDPhexose 3-C-methyltransferase I.

\section{Competing interests}

The authors declare that they have no competing interests.

\section{Authors' contributions}

LS, SJ were responsible for planning and designing the study; GFR carried out the sequence alignments; GFR, LS, and JAM were responsible for data analysis and manuscript preparation. All authors read and approved the final manuscript.

\section{Acknowledgements}

Part of the cost of this study was supported in part by the following grants: 2/0156/11 of the Scientific Grant Agency of the Ministry of Education of the Slovak Republic and the Slovak Academy of Sciences the 26240120030 (TRANSMED2), and 26240220062 (Center for innovative research of anticancer and antiviral strategies) supported by the Research \& Development Operational Programme funded by the ERDF.

\section{Author details}

'Department of Rickettsiology, Institute of Virology, Slovak Academy of Sciences, Dubravska cesta, 9, Bratislava 845 05, Slovakia. ${ }^{2}$ Laboratory of Protein Evolution, Institute of Molecular Biology, Slovak Academy of Sciences, Bratislava 845 51, Slovakia. ${ }^{3}$ USDA, Agricultural Research Service, Plant Genetics Research Unit, Columbia, MO 65211, USA. ${ }^{4}$ Interdisciplinary Plant Group, University of Missouri, Columbia, MO 65211, USA ${ }^{5}$ Department of Biochemistry, University of Missouri, Columbia, MO 65211, USA. ${ }^{6}$ Centre for Molecular Medicine, Slovak Academy of Sciences, Bratislava 831 01, Slovakia.

Received: 25 June 2012 Accepted: 5 November 2012 Published: 15 November 2012

\section{References}

1. Ghigo E, Colombo Ml, Heinzen RA: The Coxiella burnetii parasitophorous vacuole. Adv Exp Med Biol 2012, 984:141-169.

2. Arricau-Bouvery $\mathrm{N}$, Rodolakis $\mathrm{A}$ : Is $\mathrm{Q}$ fever an emerging or re-emerging zoonosis? Vet Res 2005, 36:327-349.

3. Tissot-Dupont H, Raoult D: Q Fever. Clin N Am 2008, 22:505-514

4. Moeller RB: Causes of caprine abortion: diagnostic assessment of 211 cases (1991-1998). J Vet Diagn Invest 2001, 13:265-270.

5. Bildfell RJ, Thomson GW, Haines DM, McEwen BJ, Smart N: Coxiella burnetii infection is associated with placentitis in cases of bovine abortion. $J$ Vet Diagn Invest 2000, 12:419-425.

6. Waldhalm DG, Stoenner HG, Simmons RE, Thomas LA: Abortion associated with Coxiella burnetii infection in dairy goats. J Am Vet Med Assoc 1978, 12:1580-1581.

7. Raoult D, Marrie T, Mege J: Natural history and pathophysiology of Q fever. Lancet Infect Dis 2005, 5:219-226.

8. Kazar J: Coxiella burnetii infection. Ann N Y Acad Sci 2005, 1063:105-114.

9. Parker NR, Barralet JH, Bell AM: Q fever. Lancet 2006, 367:679-688.

10. Marrie TJ: Coxiella burnetii pneumonia. Eur Respir J 2003, 21:713-719.

11. Woldehiwet Z: Q fever (coxiellosis): epidemiology and pathogenesis. Res Vet Sci 2004, 77:93-100.

12. Palkovicova $K$, Ihnatko R, Vadovic P, Betinova E, Skultety L, Frangoulidis D, Toman R: A monoclonal antibody specific for a unique biomarker virenose in a lipopolysaccharide of Coxiella burnetii. Clin Microbiol Infect 2009, 2:183-194.

13. Toman R, Skultety L, Ihnatko R: Coxiella burnetii glycomics and proteomics-tools for linking structure to function. Ann N Y Acad Sci 2009 1166:67-78.

14. Hoover TA, Culp DW, Vodkin MH, Williams JC, Thompson HA: Chromosomal DNA deletions explain phenotypic characteristics of two antigenic variants phase II and RSA 514 (crazy) of the Coxiella burnetii nine mile strain. Infect Immun 2002, 70:6726-6733.

15. Skultety $L$, Toman R, Patoprsty $V$ : A comparative study of lipopolysaccharides from two Coxiella burnetii strains considered to be associated with acute and chronic Q fever. Carbohyd Polymers 1998, 35:189-194.

16. Toman R, Skultety L: Structural study on a lipopolysacharide from Coxiella burnetii strain Nine Mile in avirulent phase II. Carbohydr Res 1996, 283:175-185.

17. Scharamek S, Radziejewska-Lebrecht J, Mayer H: 3-C-Branched aldoses in lipopolysacharide of phase I Coxiella burnetii and their role as immunodominant factors. Eur J Biochem 1985, 148:455-461.

18. Stocker MGP. Fiset $P$ : Phase variation of the Nine Mile and other strains of Rickettsia burneti. Can J Microbiol 1956, 2:310-321.

19. Slaba K, Skultety L, Toman R: Efficiency of various serological techniques for diagnosing Coxiella burnetii infection. Acta Virol 2005, 49:123-127.

20. Denison AM, Massung RF, Thompson HA: Analysis of the O-antigen biosynthesis regions of phase II isolates of Coxiella burnetii. FEMS Microbiol Lett 2007, 267:102-107.

21. Skultety L, Hajduch M, Florez-Ramirez G, Miernyk JA, Ciampor F, Toman R, Sekeyova Z: Proteomic comparison of virulent phase I and avirulent phase II of Coxiella burnetii, the causative agent of Q fever. J Proteom 2011, 74:1974-1984.

22. Thibodeaux CH, Melancon CE, Liu HW: Unusual sugar biosynthesis and natural product glycodiversification. Nature 2007, 446:1008-1016.

23. Salas JA, Méndez C: Biosynthesis pathways for deoxysugars in antibiotic-producing actinomycetes: isolation, characterization and generation of novel glycosylated derivatives. J Molec Microbiol Biotechnol 2005, 9:77-85.

24. Liu B, Knirel YA, Feng L, Perepelov AV, Senchenkova SN, Wang Q, Reeves PR, Wang L: Structure and genetics of Shigella $O$ antigens. FEMS Microbiol ReV 2008, 32:627-653.

25. He XM, Liu HW: Formation of unusual sugars: mechanistic studies and biosynthetic applications. Annu Rev Biochem 2002, 71:701-754.

26. Seshadri R, Paulsen IT, Eisen JA, Read TD, Nelson KE, Nelson WC, Ward NL, Tettelin H, Davidsen TM, Beanan MJ, Deboy RT, Daugherty SC, Brinkac LM, Madupu R, Dodson RJ, Khouri HM, Lee KH, Carty HA, Scanlan D, Heinzen RA, Thompson HA, Samuel JE, Fraser CM, Heidelberg JF: Complete genome sequence of the Q-fever pathogen Coxiella burnetii. Proc Natl Acad SC USA 2003, 100:5455-5460.

27. Hackstadt T, Williams JC: Biochemical stratagem for obligate parasitism of eukaryotic cells by Coxiella burnetii. Proc Natl Acad Sci USA 1981, 78:3240-3244

28. Patetsky D, Consigli RA, Downs CM: Studies on the physiology of rickettsiae III. Glucose phosphorylation and hexokinase activity in Coxiella burnetii. J Bacteriol 1962, 83:538-543.

29. Beare PA, Unsworth N, Andoh M, Voth DE, Omsland A, Gilk SD, Williams KP Sobral BW, Kupko JJ, Porcella SF, Samuel JE, Heinzen RA: Comparative genomics reveal extensive transposon-mediated genomic plasticity and diversity among potential effector proteins within the genus Coxiella. Infect Immun 2009, 77:642-656.

30. Proudfoot AEl, Turcatti G, Wells TNC, Payton MA, Smith DJ: Purification, cDNA cloning and heterologous expression of human phosphomannose isomerase. Eur J Biochem 1994, 219:415-423.

31. Darzins A, Frantz B, Vanags RI, Chakrabarty AM: Nucleotide sequence analysis of the phosphomannose isomerase gene (PMI) of Pseudomonas aeruginosa and comparison with the corresponding Escherichia coli gene manA. Gene 1986, 42:293-302.

32. Köplin R, Arnold W, Hötte B, Simon R, Wang G, Pühler A: Genetics of xanthan production in Xanthomonas campestris: the xan $A$ and $x a n B$ genes are involved in UDP-glucose and GDP-mannose biosynthesis. J Bacteriol 1992, 174:191-199.

33. Lee HJ, Chang HY, Venkatesan N, Peng HL: Identification of amino acids residues important for the phosphomannose isomerase activity of PsIB in Pseudomonas aeroginosa PAO1. FEBS Lett 2008, 582:3479-3483.

34. Jensen SO, Reeves PR: Molecular evolution of the GDP-mannose pathway genes (manB and manC) in Salmonella enteric. Microbiology 2001, 147:599-610.

35. Regni C, Naught L, Tipton PA, Beamer LJ: Structural basis of diverse substrate recognition by the enzyme PMM/PGM from $P$ aeruginosa. Structure 2004, 12:55-63. 
36. Takahashi H, Liu Y-n, Liu HW: A two-stage one pot enzymatic synthesis of TDP-L-mycarose from thymidine and glucose-1-phosphate. J Am Chem Soc 2006, 128:1432-1433.

37. Blankenfeldt W, Asuncion M, Lam JS, Naismith JH: The structural basis of the catalytic mechanism and regulation of the glucose-1-phosphate thymidylyltransferase (RmIA). EMBO J 2000, 19:6652-6663.

38. Lindquist $L$, Kaiser $R$, Reeves $P$, Lindberg A: Purification characterization and HPLC assay of Salmonella glucose-1-phosphate thymidylyltransferase from the cloned rfbA gene. Eur J Biochem 2005, 211:763-770.

39. Summers RG, Donadio S, Staver MJ, Wendt-Pienkowski E, Hutchinson CR, Katz $L$ : Sequencing and mutagenesis of genes from the erythromycin biosynthetic gene cluster of Saccharopolyspora erythraea that are involved in L-mycarose and D-desosamine production. Microbiology 1997, 143:3251-3262.

40. Alm RA, Ling LS, Moir DT, King BL, Brown ED, Doing PC, Smith DR, Noonan B, Guild BC, De Jonge B, Carmel G, Tummino PJ, Caruso A, Uria-Nickelsen M, Mills DM, Ives C, Gibson R, Merberg D, Mills SD, Jiang Q, Taylor DE, Vovis GF, Trust TJ: Genomic-sequence comparison of two unrelated isolates of the human gastric pathogen Helicobacter pylori. Nature 1999, 397:176-180.

41. Allard ST, Giraud MF, Whitfield C, Graninger M, Messner P, Naismith JH: The crystal structure of dTDP-D-Glucose 46-dehydratase (RmIB) from Salmonella enterica serovar Typhimurium the second enzyme in the dTDP-I-rhamnose pathway. J Mol Biol 2001, 307:283-295.

42. Ren $Y$, Perepelov Al, Wang $H$, Zhang $H$, Knirel YA, Wang $L$, Chen W: Biochemical characterization of GDP-L-fucose de novo synthesis pathway in fungus Mortierella alpine. BBRC 2010, 391:1663-1669.

43. Webb NA, Mulichak AM, Lam JS, Rocchetta HL, Garavito RM: Crystal structure of a tetrameric GDP-d-mannose 46-dehydratase from a bacterial GDP-d-rhamnose biosynthetic pathway. Protein Sci 2004, 13:529-539

44. Somoza JR, Menon SW, Somers WS, Sullivan F: Structural and kinetic analysis of Escherichia coli GDP-mannose 46 dehydratase provides insights into the enzyme's catalytic mechanism and regulation by GDPfucose. Structure 2000, 8:123-135.

45. Chen H, Zhao Z, Hallis TM, Guo Z, Liu HH: Insights into the branchedchain formation of mycarose: Methylation catalyzed by an (S) adenosylmethionine-dependent methyltransferase. Angew Chem Int Ed 2001, 40:607-610.

46. Gaisser S, Böhm GA, Doumith M, Raynal MC, Dhillon N, Cortés J, Leadlay PF: Analysis of eryBI eryBIII and eryBVII from the erythromycin biosynthetic gene cluster in Saccharopolyspora erythraea. Mol Gen Genet 1998, 258:78-88.

47. Weitnauer G, Gaisser S, Kellenberger L, Leadlay PF, Bechthold A: Analysis of a C-methyltransferase gene (aviG1) involved in avilamycin biosynthesis in Streptomyces viridochromogenes Tu57 and complementation of a Saccharopolyspora erythraea eryBIII mutant by aviG1. Microbiology 2002, 148:373-379.

48. Schluckebier G, O'Gara M, Saenger W, Cheng X: Universal catalytic domain structure of AdoMet-dependent methyltransferases. J Mol Biol 1995, 247:16-20.

49. Boissier F, Bardou V, Guillet S, Uttenwiler-Joseph M, Daffe A, Quemard A, Mourey L: Further insight into S-adenosylmethionine-dependent methyltransferases: structural characterization of Hma an enzyme essential for the biosynthesis of oxygenated mycolic acids in Mycobacterium tuberculosis. J Biol Chem 2006, 281:4434-4445

50. Gaisser S, Böhm GA, Cortés J, Leadlay PF: Analysis of seven genes from the eryAl-eryK region of the erythromycin biosynthetic gene cluster in Saccharopolyspora erythraea. Mol Gen Genet 1997, 256:239-251.

51. Bate N, Butler AR, Smith IP, Cundliffe E: The mycarose-biosynthetic genes of Streptomyces fradiae producer of tylosin. Microbiology 2000, 146:139-146.

52. Perez M, Baig I, Brana AF, Salas JA, Rohr J, Mendez C: Generation of new derivatives of the antitumor antibiotic mithramycin by altering the glycosylation pattern through combinatorial biosynthesis. Chem BioChem 2008, 9:2295-2304

53. Gonzalez A, Remsing LL, Lombo F, Fernandez MJ, Prado L, Braña AFE, Kunzel EJ, Rohr J, Méndez C, Salas JA: The mtmVUC genes of the mithramycin gene cluster in Streptomyces argillaceus are involved in the biosynthesis of the sugar moieties. Mol Gen Genet 2001, 264:827-835.
54. Yamase H, Zhao L, Liu HW: Engineering a hybrid sugar biosynthetic pathway: production of I-rhamnose and its implication on dihydrostreptose biosynthesis. J Am Chem Soc 2000, 122:12397-12398.

55. King JD, Poon KK, Webb NA, Anderson EM, McNally DJ, Brisson JR, Messner P, Garavito RM, Lam JS: The structural basis for catalytic function of GMD and RMD two closely related enzymes from the GDP-D-rhamnose biosynthesis pathway. FEBS J 2009, 276:2686-2700.

56. Kneidinger B, Graninger M, Adam G, Puchberger M, Kosma P, Zayni S, Messner P: Identification of two GDP-6-deoxy-d-lyxo-4-hexulose reductases synthesizing GDP-d-rhamnose in Aneurinibacillus thermoaerophilus L420-91T. J Biol Chem 2001, 276:5577-5583.

57. Tonetti M, Zanardi D, Gurnon JR, Fruscione F, Armirotti A, Damonte G, Sturla L, De Flora A, Van Etten JL: Paramecium Bursaria chlorella virus 1 encodes two enzymes involved in the biosynthesis of GDP-L-fucose and GDP-Drhamnose. J Biol Chem 2003, 278:21559-21565.

58. Yamamoto K, Katayama I, Onoda Y, Inami M, Kumagai H, Tochikura T: Evidence that the enzyme catalyzing the conversion of guanosine diphosphate D-mannose to a 4-keto sugar nucleotide intermediate requires nicotinamide adenine dinucleotide phosphate. Arch Biochem Biophys 1993, 300:694-698

59. Lau STB, Tanner ME: The mechanism and active site residues of GDPfucose synthase. J Am Chem Soc 2008, 130:17593-17602.

60. Rosano C, Bisso A, Izzo G, Tonetti M, Sturla L, De Flora A, Bolognesi M: Probing the catalytic mechanism of GDP-4-keto-6-deoxy-d-mannose epimerase/reductase by kinetic and crystallographic characterization of site-specific mutants. J Mol Biol 2000, 303:77-91.

61. Narasaki CT, Mertens K, Samuel JE: Characterization of the GDP-mannose biosynthesis pathway in Coxiella burnetii: The initial steps for GDP- $\beta$-DVirenose biosynthesis. PLoS One 2011, 6:e25514.

62. Consortium UP: Ongoing and future developments at the Universal Protein Resource. Nucl Acids Res 2011, 39:D214-D219.

63. Benson DA, Karsch-Mizrachi I, Lipman DJ, Ostell J, Sayers EW: GeneBank. Nucl Acids Res 2011, 39:D32-D37.

64. Altschul SF, Gish W, Miller W, Myers EW, Lipman DJ: Basic local alignment search tool. J Mol Biol 1990, 215:403-410.

65. Chenna R, Sugawara H, Koike T, Lopez R, Gibson TJ, Higgins DG, Thompson JD: Multiple sequence alignment with the Clustal series of programs. Nucl Acids Res 2003, 31:3497-3500.

66. Rose PW, Beran B, Bi C, Bluhm WF, Dimitropoulos D, Goodsell DS, Prlic A, Quesada M, Quinn GB, Westbrook JD, Young J, Yukich B, Zardecki C, Berman HM, Bourne PE: The RCSB protein data bank: redesigned web site and web services. Nucl Acids Res 2011, 39:D392-D401.

\section{doi:10.1186/1477-5956-10-67}

Cite this article as: Flores-Ramirez et al: In silico biosynthesis of virenose, a methylated deoxy-sugar unique to Coxiella burnetii lipopolysaccharide. Proteome Science 2012 10:67.

\section{Submit your next manuscript to BioMed Central and take full advantage of:}

- Convenient online submission

- Thorough peer review

- No space constraints or color figure charges

- Immediate publication on acceptance

- Inclusion in PubMed, CAS, Scopus and Google Scholar

- Research which is freely available for redistribution 